\title{
SEIFERT FIBERED SURGERY MANIFOLDS OF COMPOSITE KNOTS
}

\author{
JOHN KALLIONGIS AND CHICHEN M. TSAU
}

(Communicated by Frederick R. Cohen)

\begin{abstract}
A classification is given for the composite knots and the Dehn surgery on these knots which yield Seifert fibered surgery manifolds. We prove that if a knot $K$ is the composition of two torus knots, then some (unique) integral surgery on $K$ yields a Seifert fibered manifold, and conversely if the surgery manifold of a composite knot $K$ is Seifert fibered, then $K$ is the composition of two torus knots and the surgery must be integral surgery, which is uniquely determined.
\end{abstract}

In this paper we classify the composite knots and the Dehn surgeries on these knots which yield Seifert fibered surgery manifolds. In [8] Moser conjectured that surgery on a nontorus knot could not yield a Seifert fibered manifold, in particular, could not yield a lens space. Many counterexamples to Moser's conjecture have been found. Baily and Rolfsen [2] showed that the lens space $L(23,7)$ could be obtained by -23 -surgery on the $(11,2)$-cable knot about the trefoil knot; Simon discovered similar examples. Fintushel and Stern [4] constructed infinitely many noniterated torus knots, upon which certain surgery yields lens spaces. More recently, Gordon [6] classified the surgery manifolds of all iterated torus knots, Berge [3] and Gabai [5] have independently constructed an infinite collection of knots in solid tori such that certain surgery on them in the solid torus yield $D^{2} \times S^{1}$, and therefore yield lens spaces when the knots are considered to be in $S^{3}$. Since cable knots are prime knots and the surgery manifold of a composite knot contains an incompressible torus (see, for example, [6]), none of the above-mentioned knots is a composite knot. Indeed, no nontrivial surgery on a composite knot may yield a lens space. It is then natural to ask if any such surgery manifold is Seifert fibered. In this paper, we show that if a knot $K$ is the composition of two torus knots, then some (unique) integral surgery on $K$ yields a Seifert fibered manifold, and conversely if a surgery manifold of a composite knot $K$ is Seifert fibered, then the surgery

Received by the editors January 4, 1989.

1980 Mathematics Subject Classification (1985 Revision). Primary 57M05, 57M25.

Key words and phrases. Composite knot, torus knot, surgery manifold, Seifert fibered manifold, incompressible surface.

The second author was supported in part by Saint Louis University Beaumont Fund. 
must be an integral surgery, $K$ must be the composition of two torus knots, and the integer is uniquely determined.

Let $m, n$ be relatively prime integers and $n \neq 0$, let $K$ be a nontrivial knot with meridian-longitude pair $(\mu, \lambda)$, and let $V$ be a solid torus with meridianlongitude $\left(\mu_{0}, \lambda_{0}\right)$. The surgery manifold $(K ; m / n)$ obtained by performing Dehn surgery of type $m / n$ on $K$ is $X \cup_{h} V$, where $h: \partial V \rightarrow \partial X$ is the gluing homeomorphism $h\left(\mu_{0}\right)=m \mu+n \lambda$. Note that up to homeomorphism of the surgery manifold, we may assume $n>0$.

Suppose $K=K_{1} \sharp K_{2}$ is the composition of the knots $K_{1}$ and $K_{2}$, and let $\left(\mu_{1}, \lambda_{1}\right),\left(\mu_{2}, \lambda_{2}\right)$ and $(\mu, \lambda)$ be the meridian-longitude pairs for $K_{1}, K_{2}$ and $K$ respectively. Denote by $X_{1}=S^{3} \backslash \stackrel{\circ}{N}\left(K_{1}\right), X_{2}=S^{3} \backslash \stackrel{\circ}{N}\left(K_{2}\right)$, and $X=$ $S^{3} \backslash \stackrel{\circ}{N}(K)$ the knot manifolds of $K_{1}, K_{2}$, and $K$ respectively. Notice that $X_{1}$, $X_{2}$, and $X$ are cubes with knotted holds (see [1]). We have $X=X_{1} \cup_{A_{1}=A_{2}} X_{2}$ where $A_{1}$ and $A_{2}$ are two meridional annuli on $\partial X_{1}$ and $\partial X_{2}$ respectively, along which $X_{1}$ and $X_{2}$ are identified. In $X$ we may assume $\mu_{1}=\mu_{2}=\mu$ and $\lambda=\lambda_{1} \sharp_{a_{1}=a_{2}} \lambda_{2}$, where $a_{1}$ and $a_{2}$ are spanning arcs in $A_{1}$ and $A_{2}$ respectively, along which $\lambda_{1}$ and $\lambda_{2}$ are identified. Writing $\lambda_{i}=\hat{\lambda}_{i} \cup_{\partial} a_{i}$ for $i=1,2$, we have $\lambda=\hat{\lambda}_{1} \cup_{\partial} \hat{\lambda}_{2}$. (See Figure 1, where $K_{1}=K_{2}$ is a trefoil knot.)

Let $p, q$ be relatively prime integers and $|p| \geq 2$ and $|q| \geq 2$. A $(p, q)$-torusknot $K_{p, q}$ is a knot lying on the boundary of an unknotted solid torus in $S^{3}$, wrapping $p$ times in the meridional direction and $q$ times in the longitudional direction. The knot manifold $S^{3} \backslash \stackrel{\circ}{N}\left(K_{p, q}\right)$ is a Seifert fibered manifold over a disk with two singular fibers of indices $q$ and $p$ respectively. Furthermore,
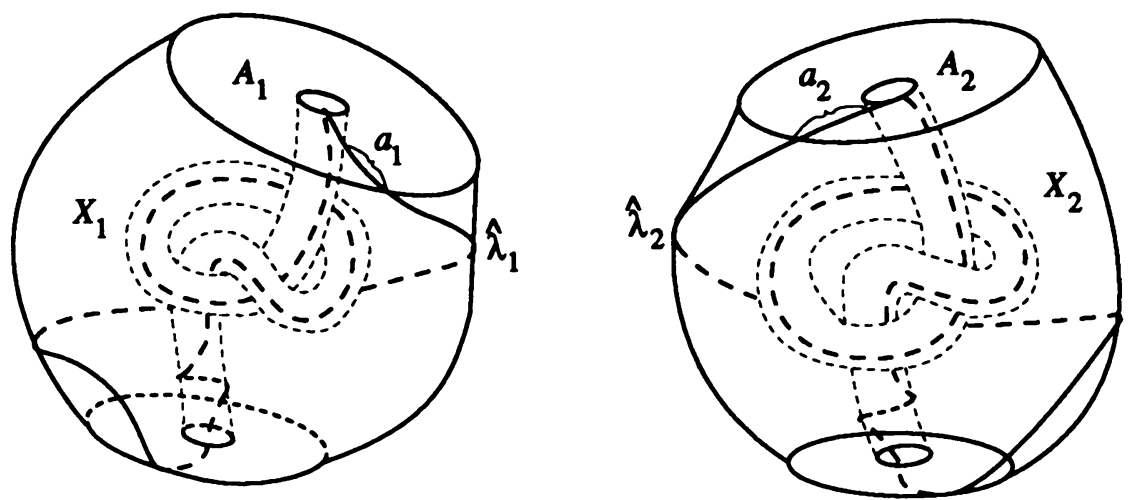

FIGURE 1. 
the Seifert fibration is unique up to fiber preserving homeomorphisms (see, for example, [7, Theorem VI 10]). A fiber in $\partial\left(S^{3} \backslash \stackrel{\circ}{N}\left(K_{p, q}\right)\right)$, when properly oriented, is a $(p q, 1)$-curve in $\partial\left(S^{3} \backslash \stackrel{\circ}{N}\left(K_{p, q}\right)\right)$ with respect to the meridianlongitude for $K_{p, q}$.

Our proofs are based on the observation (see the remark after [6, Lemma 7.1]) that if an integral surgery $m$ is performed on a composite knot $K_{1} \sharp K_{2}$, then $\left(K_{1} \sharp K_{2} ; m\right)$ is homeomorphic to $X_{1} \cup_{\partial} X_{2}$. In addition, we use the characterization theorem [7, Theorem VI 3.4]) of 2-sided incompressible surfaces embedded in Seifert fibered manifolds.

Suppose $K=K_{1} \sharp K_{2}$ and consider $(K ; m / n)$. Then the glueing homeomorphism $h: \partial V \rightarrow \partial X$ satisfies $h\left(\mu_{0}\right)=m \mu+n \lambda$ and $h\left(\lambda_{0}\right)=c \mu+d \lambda$, where $c, d$ is a pair of integers satisfying $m d-n c=1$. Therefore, $h^{-1}(\mu)=d \mu_{0}-n \lambda_{0}$. The choice of the pair $c, d$ is not unique, up to homeomorphism of the surgery manifold. Therefore, if $n=1$, we may choose $d=0$ and $c=-1$, so that $h\left(\lambda_{0}\right)=-\mu$. Then $h^{-1}(\mu)=-\lambda_{0}$, which implies a meridional annulus in $\partial X$ is glued to a longitudional annulus in $\partial V$, via $h$. Now from the proof of [6, Lemma 7.1] we have $(K ; m) \cong\left(X_{1} \cup_{B_{1}=B_{0}} V\right) \cup_{\partial} X_{2}$, where $B_{1}$ is the meridional annulus $\partial X_{1} \backslash \stackrel{\circ}{A}$ contained in $\partial X$ on $\partial X_{1}, B_{0}=h^{-1}\left(B_{1}\right)$, and so $B_{0}$ is a longitudional annulus on $\partial V$. This implies that $X_{1} \cup_{B_{1}=B_{0}} V$ is obtained from $X_{1}$ by capping the solid torus $V$ to $X_{1}$ on the boundary along the annulus $B_{1}$. Hence $X_{1} \cup_{B_{1}=B_{0}} V=X_{1}$ and therefore $(K ; m)=X_{1} \cup_{\partial} X_{2}$. Also the proof of [6, Lemma 7.1] showed that $\partial\left(X_{1} \cup_{B_{1}=B_{0}} V\right)=\partial X_{2}$ is a 2-sided separating incompressible torus in $(K ; m / n)$ and that $B_{1}=B_{0}$ is a 2-sided incompressible properly embedded annulus in $X_{1} \cup_{B_{1}=B_{0}} V$.

For completeness we include the following characterization theorem for 2sided incompressible surfaces in Seifert fibered manifolds mentioned above ([7, Theorem VI 3.4]).

Theorem 0. Let $M$ be a compact, orientable Seifert fibered manifold. If $F$ is a two-sided incompressible surface in $M$, then one of the following alternatives holds:

(i) $F$ is a disk or an annulus and $F$ is parallel into $\partial M$.

(ii) $F$ does not separate $M$ and $F$ is a fiber in a fibration of $M$ as a surface bundle over $S^{1}$.

(iii) $F$ does separate $M$ and $M=M_{1} \cup M_{2}$ where $M_{1} \cap M_{2}=\partial M_{1}=$ $\partial M_{2}=F$ and $M_{i}(i=1,2)$ is a twisted I-bundle over a compact surface (possibly with boundary).

(iv) $F$ is an annulus or a torus and $F$ is saturated in some Seifert fibration of $M$.

The following well-known lemmas and corollary will be needed in the proof of Theorem 4. For completeness we supply the proofs. 
Lemma 1. If $K$ is a nontrivial knot, then $S^{3} \backslash \stackrel{\circ}{N}(K)$ is not a twisted I-bundle over a compact surface (with or without boundary).

Proof. Assume $S^{3} \backslash \stackrel{\circ}{N}(K)$ is a twisted $I$-bundle over a compact surface $F$ of genus $g$. If $\partial F=\varnothing$, then $H_{1}\left(S^{3} \backslash \stackrel{\circ}{N}(K)\right)=H_{1}(F)$, which equals $\mathbf{Z}^{2 g}$ if $F$ is orientable, and equals $\mathbf{Z}^{g-1}+\mathbf{Z}_{2}$ if $F$ is nonorientable. However $H_{1}\left(S^{3} \backslash \stackrel{\circ}{N}(K)\right)=\mathbf{Z}$, which gives a contradiction. If $\partial F \neq \varnothing$, then being a twisted $I$-bundle over $F, S^{3} \backslash \stackrel{\circ}{N}(K)$ should contain a Mobius band in the boundary, which is impossible.

Lemma 2. If $K$ is a nontrivial knot, then $S^{3} \backslash \stackrel{\circ}{N}(K)$ cannot be Seifert fibered without singular fibers.

Proof. Suppose $S^{3} \backslash \stackrel{\circ}{N}(K)$ is Seifert fibered over a surface $F$ of genus $g$ without singular fibers, and hence a fiber bundle. Then if $F$ is orientable,

$H_{1}\left(S^{3} \backslash \stackrel{\circ}{N}(K)\right)=\left(\left(\sum_{i=1}^{g}(\mathbf{Z}+\mathbf{Z})\right)+\mathbf{Z} d+\mathbf{Z} h\right) /\left(h^{\beta}=d\right)=\left(\sum_{i=1}^{g}(\mathbf{Z}+\mathbf{Z})\right)+\mathbf{Z}_{h}$.

Since $H_{1}\left(S^{3} \backslash \stackrel{\circ}{N}(K)\right)=\mathbf{Z}$, it follows that $g=0$ and hence $F$ is a disk. This implies $S^{3} \backslash \stackrel{\circ}{N}(K)$ is homeomorphic to a solid torus, and therefore $K$ is a trivial knot, giving a contradiction. If $F$ is nonorientable, then $\left.H_{1}\left(S^{3} \backslash \stackrel{\circ}{N}(K)\right)\right)=$ $\left(\sum_{i=1}^{g} \mathbf{Z}\right)+\mathbf{Z}_{2}$, which is not isomorphic to $\mathbf{Z}$, again giving a contradiction. This shows $S^{3} \backslash \stackrel{\circ}{N}(K)$ cannot be Seifert fibered without singular fibers, completing the proof.

Applying Lemma 2 and [8, Theorem 2] we obtain

Corollary 2.1. If the knot manifold of a nontrivial knot is Seifert fibered, then the knot must be a torus knot.

We now state and prove our main results.

Theorem 3. If $K=K_{p, q} \sharp K_{r, s}$ is the composition of torus knots $K_{p, q}$ and $K_{r, s}$, then the surgery manifold $(K ; p q+r s)$ is a Seifert fibered manifold over $S^{2}$ with four exceptional fibers of indices $q, p, s$, and $r$.

Proof. Let $\left(\mu_{1}, \lambda_{1}\right),\left(\mu_{2}, \lambda_{2}\right)$ and $(\mu, \lambda)$ be the meridian-longitude pairs for $K_{p, q}, K_{r, s}$, and $K$ respectively, and let $X_{1}=S^{3} \backslash \stackrel{\circ}{N}\left(K_{p, q}\right), X_{2}=S^{3} \backslash \stackrel{\circ}{N}\left(K_{r, s}\right)$ and $X=S^{3} \backslash \stackrel{\circ}{N}(K)$. Then both $X_{1}$ and $X_{2}$ are Seifert fibered over a disk with two singular fibers of indices $q, p$ and $r, s$ respectively. Assuming the previous notations, we have $h\left(\mu_{0}\right)=(p q+r s) \mu+\lambda, h^{-1}(\mu)=\lambda_{0}$, and $(K ; p q+r s)=$ $\left(X_{1} \cup_{B_{1}=B_{0}} V\right) \cup_{\partial} X_{2}$. We will show that $\left(X_{1} \cup_{B_{1}=B_{0}} V\right)$ can be Seifert fibered and the fibers on $\partial\left(X_{1} \cup_{B_{1}=B_{0}} V\right)$ are glued to the fibers on $\partial X_{1}$ via the identification map. 
Now $h\left(\mu_{0}\right)=(p q+r s) \mu+\lambda$ is a $(p q+r s, 1)$ curve on $\partial X$. Since $\mu_{1}=$ $\mu_{2}=\mu, \lambda=\lambda_{1} \sharp_{a_{1}=a_{2}} \lambda_{2}$ and $\lambda_{i}=\hat{\lambda}_{i} \cup_{\partial} a_{i}$ for $i=1,2$, by replacing $\mu_{0}$ with another meridian of $V$ if necessary, we may assume $h\left(\mu_{0}\right)=(p q+r s) \mu+\lambda=$ $\left(p q \mu_{1}+\lambda_{1}\right) \sharp_{a_{1}=a_{2}}\left(r s \mu_{2}+\lambda_{2}\right)=\left(p q \mu_{1}+\hat{\lambda}_{1}\right) \cup_{\partial}\left(r s \mu_{2}+\hat{\lambda}_{2}\right)$, where $p q \mu_{1}+\hat{\lambda}_{1}$ is the arc $\left(p q \mu_{1}+\lambda_{1}\right) \backslash \stackrel{\circ}{a}_{1}$ contained in $B_{1}$ and $r s \mu_{2}+\hat{\lambda}_{2}$ is the arc $\left(r s \mu_{2}+\lambda_{2}\right) \backslash \stackrel{\circ}{a}_{2}$ contained in $\partial X_{2} \backslash \stackrel{\circ}{A}$ (see Figure 2). Let $\mu_{0}^{(1)}=h^{-1}\left(p q \mu_{1}+\lambda_{1}\right)$ and $\mu_{0}^{(2)}=h^{-1}\left(r s \mu_{2}+\lambda_{2}\right)$. Then $\mu_{0}=\mu_{0}^{(1)} \cup_{\partial} \mu_{0}^{(2)}$ and $h\left(\mu_{0}^{(1)}\right)=p q \mu_{1}+\hat{\lambda}_{1}$ and $h\left(\mu_{0}^{(2)}\right)=r s \mu_{2}+\hat{\lambda}_{2}$. Since $h\left(\mu_{0}^{(1)}\right) \cup_{\partial} a_{1}=p q \mu_{1}+\lambda_{1}$ is a $(p q, 1)$-curve on $\partial X_{1}$, it is freely homotopic to a fiber in $\partial X_{1}$, and therefore we may refiber $X_{1}$, if necessary, so that $h\left(\mu_{0}^{(1)}\right) \cup_{\partial} a_{1}$ is a fiber in $\partial X_{1}$. Since $f\left(\mu_{0}^{(1)}\right)$ is freely isotopic (rel $\partial$ ) to $\mu_{0}^{(2)}$ in $X_{1} \cup_{B_{1}=B_{0}} V$, and $X_{1} \cup \cup_{B_{1}=B_{2}} V=X_{1}$, it follows that $X_{1} \cup_{B_{1}=B_{2}} V$ can be Seifert fibered so that $\mu_{0}^{(1)} \cup_{\partial} a_{1}$ is a fiber on $\partial\left(X_{1} \cup_{B_{1}=B_{0}} V\right)$. Now $\mu_{0}^{(2)} \cup a_{1}$ is identified via $h$ with $\left(r s \mu+\hat{\lambda}_{2}\right) \cup_{\partial} a_{2}=r s \mu_{2}+\lambda_{2}$, a $(r s, 1)$-curve on $\partial X_{2}$, and therefore is homotopic to a fiber in $\partial X_{2}$. We may refiber $X_{2}$ if necessary, so that $\left(r s \mu_{2}+\hat{\lambda}_{2}\right) \cup_{\partial} a_{2}$ is a fiber in $\partial X_{2}$. Thus $(K ; p q+r s)=\left(X_{1} \cup_{B_{1}=B_{0}} V\right) \cup_{\partial} X_{2}$ is Seifert fibered. From the proof it is clear that $\left(X_{1} \cup_{B_{1}=B_{0}} V\right)$ is fibered over a disk with two singular fibers of indices $q$ and $p$; therefore $(K ; p q+r s)$ is Seifert fibered over $S^{2}$ with singular fibers of indices $q, p, s$, and $r$.

Theorem 4. Let $K_{1}$ and $K_{2}$ be nontrivial knots in $S^{3}$ and let $K=K_{1} \sharp K_{2}$ be the composition of $K_{1}$ and $K_{2}$. If the surgery manifold $(K ; m / n)$ is Seifert fibered, then $n=1$ and both $K_{1}$ and $K_{2}$ are torus knots. Furthemore, if $K_{1}=K_{p, q}$ and $K_{2}=K_{r, s}$, then $m=p q+r s$.

Proof. As stated previously, the torus $F=\partial\left(X_{1} \cup_{B_{1}=B_{0}} V\right)=\partial X_{2}$ is 2-sided, separating and incompressible in $(K ; m / n)=\left(X_{1} \cup_{B_{1}=B_{0}} V\right) \cup_{\partial} X_{2}$. By Theorem 0 and Lemma 1 (applying to $X_{2}$ ), we conclude that $F$ is a union of fibers in some Seifert fibration of $(K ; m / n)$, and therefore $X_{1} \cup_{B_{1}=B_{0}} V$ and $X_{2}$ are Seifert fibered. Since $X_{2}$ is Seifert fibered, by Corollary $2.1, K_{2}$ is a torus knot.

Now consider the Seifert fibered 3-manifold $X_{1} \cup_{B_{1}=B_{0}} V$, and let $B=B_{1}=$ $B_{0}$. Then as stated previously, $B$ is a 2-sided separting incompressible annulus in $X_{1} \cup_{B-1=B_{0}} V$. Suppose, for contradiction, $n \neq 1$. Then, since $h^{-1}(\mu)=$ $d \mu_{0}-n \lambda_{0}$, which is a $(d,-n)$ curve on $\partial V$ and is the core of the annulus $B_{0}$, it follows that $B_{0}$ is not parallel into $\partial V \backslash \stackrel{\circ}{B}_{0}$. Since $B_{1}$ is a meridional annulus on $\partial X_{1}, B_{1}$ is not parallel into $\partial X_{1} \backslash \stackrel{\circ}{B}_{1}=A_{1}$. This implies $B$ is not parallel into $\partial\left(X_{1} \cup_{B_{1}=B_{0}} V\right)=F$. By Theorem 0 and Lemma 1 (applying to $X_{1}$ ), we conclude that the annulus $B\left(=B_{1}=B_{0}\right)$ is a union of fibers in some Seifert fibration of $X_{1} \cup_{B_{1}=B_{0}} V$. This implies that both $X_{1}$ and $V$ are Seifert fibered. Now since $X_{1}$ is Seifert fibered, by Corollary $2.1, K_{1}$ is a torus knot $K_{p, q}$ for some $p$ and $q$. Then by the uniqueness of the Seifert fibration of $S^{3} \backslash \stackrel{\circ}{N}\left(K_{p, q}\right)$, 


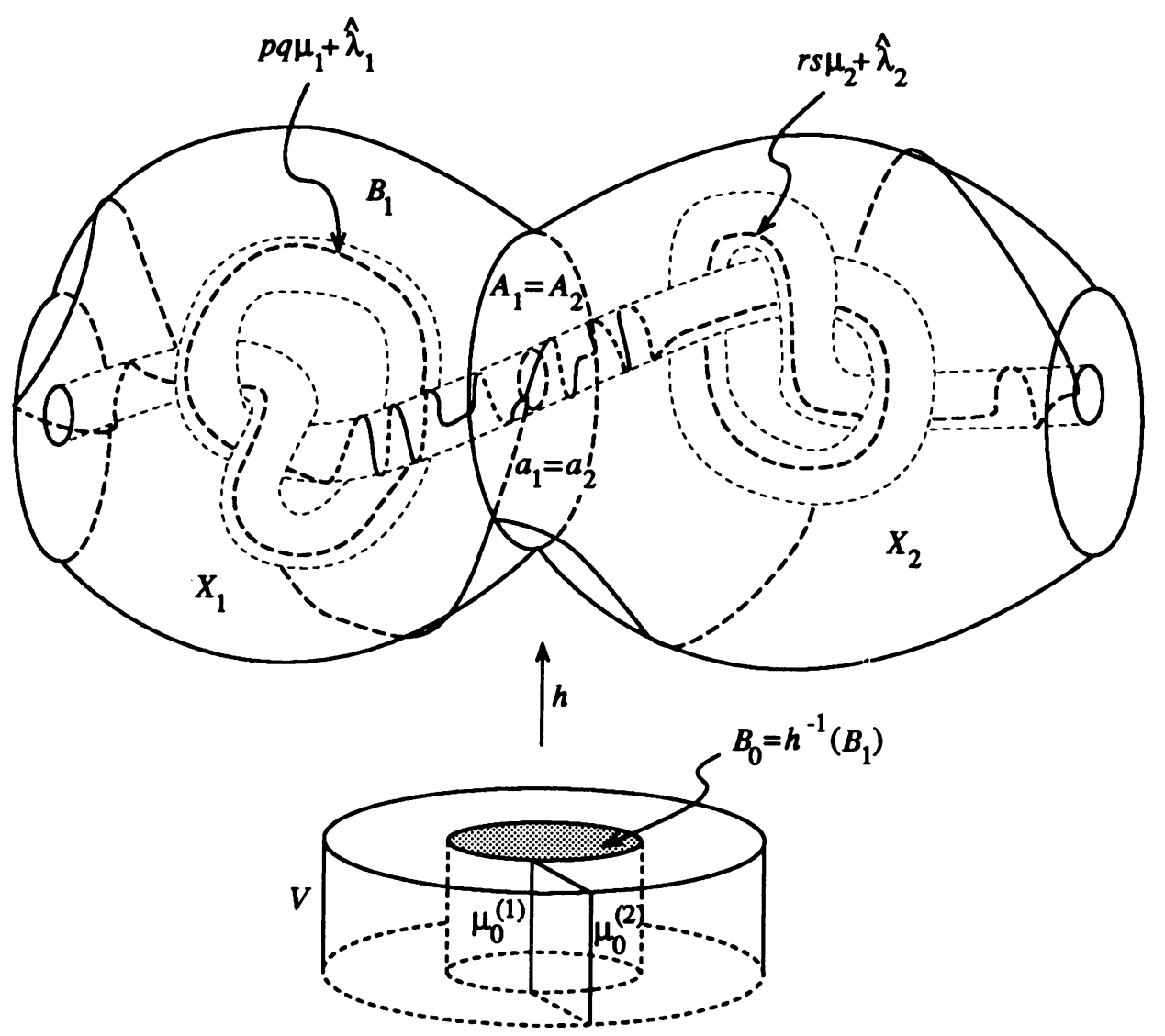

FIGURE 2.

a fiber in $\partial\left(S^{3} \backslash \stackrel{\circ}{N}\left(K_{p, q}\right)\right)=\partial X_{1}$ is a $(p q, 1)$-curve. Since $B_{1} \subset \partial X_{1}$, every fiber in $B_{1}=B$ must be a $(p q, 1)$-curve on $\partial X_{1}$. But every fiber in a fibered annulus is a generating simple closed curve, and $B_{1}$ is a meridional annulus contained in $\partial X_{1}$, hence we obtain a contradiction. Therefore, $n=1$.

Now since $n=1, X_{1} \cong X_{1} \cup_{B_{1}=B_{0}} V$; since $X_{1} \cup_{B_{1}=B_{0}} V$ is Seifert fibered, $X_{1}$ is Seifert fibered and so $K_{1}$ is a torus knot by Corollary 2.1. Let $K_{1}=K_{p, q}$ and $K_{2}=K_{r, s}$; because the Seifert fibrations of the knot manifolds $X_{1} \stackrel{p, q}{=}$ $S^{3} \backslash \stackrel{\circ}{N}\left(K_{p, q}\right)$ and $X_{2}=S^{3} \backslash \stackrel{\circ}{N}\left(K_{r, s}\right)$ are unique, a fiber in $\partial X_{1}$ is a $(p q, 1)$-curve and a fiber in $\partial X_{2}$ is a $(r s, 1)$-curve. Now $h\left(\mu_{0}\right)=m \mu+\lambda$ is an $(m, 1)$-curve in $\partial X$ where $X=X_{1} \cup \cup_{A_{1}=A_{2}} X_{2}$. By replacing $\mu_{0}$ with another meridian of $V$, if necessary, we may assume $h\left(\mu_{0}\right)=m \mu+\lambda=p q \mu+(m-p q) \mu+\lambda=$ $\left.\left(p q \mu_{1}+\lambda_{1}\right) \sharp_{a_{1}=a_{2}}\left((m-p q) \mu_{2}+\lambda_{2}\right)=\left(p q \mu_{1}+\hat{\lambda}_{1}\right) \cup_{\partial}(m-p q) \mu_{2}+\hat{\lambda}_{2}\right)$, where 
$p q \mu_{1}+\hat{\lambda}_{1}$ is the arc $(p q \mu+\lambda) \backslash \stackrel{\circ}{a}_{1}$ in $B_{1}=\partial X_{1} \backslash \stackrel{\circ}{A}_{1}$, and $(m-p q) \mu_{2}+\hat{\lambda}_{2}$ is the arc $\left(p q \mu_{2}+\lambda_{2}\right) \backslash \stackrel{\circ}{a}_{2}$ in $\partial X_{2} \backslash \stackrel{\circ}{A}_{2}$. Let $\mu_{0}^{(1)}=h^{-1}\left(p q \mu_{1}+\hat{\lambda}_{1}\right)$ and $\mu_{0}^{(2)}=$ $h^{-1}\left((m-p q) \mu_{2}+\hat{\lambda}_{2}\right)$. Then $h\left(\mu_{0}^{(1)}\right) \cup_{\partial} a_{1}=p q \mu_{1}+\lambda_{1}$ is freely homotopic to a fiber in $\partial X_{1}$. Since $X_{1} \cup_{B_{1}=B_{0}} V=X_{1}$, the Seifert fibration of $X_{1} \cup_{B_{1}=B_{0}} V$ is determined by $X_{1}$ and is unique. Now because $h\left(\mu_{0}^{(1)}\right)$ is isotopic (rel $\partial$ ) to $\mu_{0}^{(2)}$ in $X_{1} \cup_{B_{1}=B_{0}} V$, we conclude that $\mu_{0} \cup_{\partial} a_{1}$ is freely homotopic to a fiber in $\partial\left(X_{1} \cup_{B_{1}=B_{0}} V\right)$ which is identified via $h$ as a fiber in $\partial X_{2}$. Thus $h\left(\mu_{0}^{(2)}\right) \cup_{\partial} a_{2}=\left((m-p q) \mu_{2}+\hat{\lambda}_{2}\right) \cup_{\partial} a_{2}$ is freely homotopic to a fiber in $\partial X_{2}$, and is therefore an $(r s, 1)$-curve in $\partial X_{2}$. Thus $m-p q=r s$ and we have $m=p q+r s$.

\section{REFERENCES}

1. R. H. Bing and J. M. Martin, Cubes with knotted holes, Trans. Amer. Math. Soc. 155 (1971), 217-331.

2. J. Bailey and D. Rolfson, An unexpected construction of a lens space, Pacific J. Math. 71 (1977), 295-298.

3. J. Berge, The knots in $D^{2} \times S^{1}$ with nontrivial Dehn surgeries, preprint.

4. R. Fintushel and R. J. Stern, Constructing lens spaces by surgery on knots, Math Z. 175 (1980) 33-51.

5. D. Gabai, Surgery on knots in solid tori, preprint.

6. C. McA. Gordon, Dehn surgery and satellite knots, Trans. Amer. Math. Soc. 275 (1983), 687708.

7. W. Jaco, Lectures on 3-manifold topology, Regional Conf. Ser. in Math., No. 43, Amer. Math. Soc., 1980.

8. L. Moser, Elementary surgery along a torus knot, Pacific J. Math. 38 (1971), 737-745.

Department of Mathematics, Saint Louis University, Saint Louis, Missouri 63103 